\title{
Some properties of an integral operator defined by convolution
}

\author{
Muhammad Arif ${ }^{1 *}$, Khalida Inayat Noor ${ }^{2}$ and Fazal Ghani ${ }^{1}$
}

* Correspondence:

marifmaths@awkum.edu.pk

'Department of Mathematics,

Abdul Wali Khan University,

Mardan, Pakistan

Full list of author information is

available at the end of the article

\section{Abstract}

In this investigation, motivated from Breaz study, we introduce a new family of integral operator using famous convolution technique. We also apply this newly defined operator for investigating some interesting mapping properties of certain subclasses of analytic and univalent functions.

2010 Mathematics Subject Classification: 30C45; 30C10.

Keywords: close-to-convex functions, convolution, integral operators

\section{Introduction}

Let $A$ denote the class of analytic function satisfying the condition $f(0)=f(0)-1=0$ in the open unit disc $\mathbb{U}=\{z:|z|<1\}$. By $S, C, S^{*}, C^{*}$, and $K$ we means the well-known subclasses of $A$ which consist of univalent, convex, starlike, quasi-convex, and close-toconvex functions, respectively. The well-known Alexander-type relation holds between the classes $C$ and $S^{*}$ and $C^{\prime \prime}$ and $K$, that is,

$$
f(z) \in C \Leftrightarrow z f^{\prime}(z) \in S^{*},
$$

and

$$
f(z) \in C^{*} \Leftrightarrow z f^{\prime}(z) \in K .
$$

It was proved in [1] that a locally univalent function $f(\mathrm{z})$ is close-to-convex, if and only if

$$
\int_{\theta_{1}}^{\theta_{2}} \operatorname{Re}\left\{1+\frac{z f^{\prime \prime}(z)}{f^{\prime}(z)}\right\} d \theta>-\pi, \quad z=r e^{i \theta},
$$

for each $r \in(0,1)$ and every pair $\theta_{1}, \theta_{2}$ with $0 \leq \theta_{1}<\theta_{2} \leq 2 \pi$.

Let $P_{k}(\xi)$ be the class of functions $p(z)$ analytic in $\mathbb{U}$ with $p(0)=1$ and

$$
\int_{0}^{2 \pi}\left|\frac{\operatorname{Re} p(z)-\xi}{1-\xi}\right| d \theta \leq k \pi, \quad z=r e^{i \theta}, k \geq 2 .
$$

This class was introduced in [2] and for $k=2, \xi=0$, the class $p_{k}(\xi)$ reduces to the class $P$ of functions with positive real part. We consider the following classes:

(C) 2012 Arif et al; licensee Springer. This is an Open Access article distributed under the terms of the Creative Commons Attribution License (http://creativecommons.org/licenses/by/2.0), which permits unrestricted use, distribution, and reproduction in any medium, provided the original work is properly cited. 


$$
\begin{aligned}
& R_{k}(\xi)=\left\{f(z) \in A: \frac{z f^{\prime}(z)}{f(z)} \in P_{k}(\xi), z \in \mathbb{U}\right\} \\
& T_{k}(\xi)=\left\{f(z) \in A: \exists g(z) \in C: \frac{f^{\prime}(z)}{g^{\prime}(z)} \in P_{k}(\xi), z \in \mathbb{U}\right\} .
\end{aligned}
$$

These classes were studied by Noor [3-5] and Padmanabhan and Parvatham [2]. Also it can easily be seen that $R_{2}(0)=S^{\prime \prime}$ and $T_{2}(0)=K$, where $S^{\prime \prime}$ and $K$ are the well-known classes of starlike and close-to-convex functions.

Using the same method as that of Kaplan [1], Noor [6] extend the result of Kaplan given in (1.1), and proved that a locally univalent function $f(z)$ is in the class $T_{k}$, if and only if

$$
\int_{\theta_{1}}^{\theta_{2}} \operatorname{Re}\left\{1+\frac{z f^{\prime \prime}(z)}{f^{\prime}(z)}\right\} d \theta>-\frac{k}{2} \pi, \quad z=r e^{i \theta},
$$

for each $r \in(0,1)$ and every pair $\theta_{1}, \theta_{2}$ with $0 \leq \theta_{1}<\theta_{2} \leq 2 \pi$

For any two analytic functions

$$
f(z)=\sum_{n=0}^{\infty} a_{n} z^{n} \text { and } g(z)=\sum_{n=0}^{\infty} b_{n} z^{n},(z \in \mathbb{U})
$$

the convolution (Hadamard product) of $f(z)$ and $g(z)$ is defined by

$$
f(z) * \mathrm{~g}(z)=\sum_{n=0}^{\infty} a_{n} b_{n} z^{n}, \quad(z \in \mathbb{U}) .
$$

Using the techniques from convolution theory many authors generalized Breaz operator in several directions, see $[7,8]$ for example. Here, we introduce a generalized integral operator $I_{n}\left(f_{i} g_{\dot{v}} h_{i}\right)(z): A^{n} \rightarrow A$ as follows

$$
I_{n}\left(f_{i}, g_{i}, h_{i}\right)(z)=\int_{0}^{z} \prod_{i=1}^{n}\left(\left(f_{i}(t) * g_{i}(t)\right)^{\prime}\right)^{\alpha_{i}}\left(\frac{h_{i}(t)}{t}\right)^{\beta_{i}} d t
$$

where $f_{i}(z), g_{i}(z), h_{i}(z) \in A$ with $f_{i}(z) * g_{i}(z) \neq 0$ and $\alpha_{i} \beta_{i} \geq 0$ for $i=1,2, \ldots, n$. The operator $I_{n}\left(f_{i} g_{i} h_{i}\right)(z)$ reduces to many well-known integral operators by varying the parameters $\alpha_{i} \beta_{i}$ and by choosing suitable functions instead of $f_{i}(z), g_{i}(z)$. For example,

(i) If we take $g_{i}(z)=\frac{z}{(1-z)}$ for all $1 \leq i \leq n$, we obtain the integral operator

$$
I_{n}\left(f_{i}, h_{i}\right)(z)=\int_{0}^{z} \prod_{i=1}^{n}\left(f_{i}^{\prime}(t)\right)^{\alpha_{i}}\left(\frac{h_{i}(t)}{t}\right)^{\beta_{i}} d t
$$

introduced in [9].

(ii) If we take $\alpha_{i}=0$ and $1 \leq i \leq n$, we obtain the integral

$$
I_{n}\left(h_{i}\right)(z)=\int_{0}^{z} \prod_{i=1}^{n}\left(\frac{h_{i}(t)}{t}\right)^{\beta_{i}} d t
$$

introduced and studied by Breaz and Breaz [10]. 
(iii) If we take $g_{i}(z)=\frac{z}{(1-z)}, \beta_{i}=0$, we obtain the integral operator

$$
I_{n}\left(f_{i}\right)(z)=\int_{0}^{z} \prod_{i=1}^{n}\left(f_{i}^{\prime}(t)\right)^{\alpha_{i}} d t
$$

introduced and studied by Breaz et al. [11].

(iv) If we take $n=1, \alpha_{1}=0$ and $\beta_{1}=1$ in (1.4), we obtain the Alexander integral operator

$$
I_{n}\left(h_{1}\right)(z)=\int_{0}^{z}\left(\frac{h_{1}(t)}{t}\right) d t
$$

introduced in [12].

(v) If we take $n=1, \alpha_{1}=0$ and $\beta_{1}=\beta$, we obtain the integral operator

$$
I_{n}\left(h_{1}\right)(z)=\int_{0}^{z}\left(\frac{h_{1}(t)}{t}\right)^{\beta} d t
$$

studied in [13].

In this article, we study the mapping properties of different subclasses of analytic and univalent functions under the integral operator given in (1.3). To prove our main results, we need the following lemmas.

Lemma 1.1 [14]. Let $f(z) \in R_{k}(\xi)$ for $k \leq 2,0 \leq \xi<1$. Then with $0 \leq \theta_{1}<\theta_{2} \leq 2 \pi$ and $z=r e^{i \theta}, r<1$,

$$
\int_{\theta_{1}}^{\theta_{2}} \operatorname{Re}\left\{\frac{z f^{\prime}(z)}{f(z)}\right\} d \theta>-\left(\frac{k}{2}-1\right)(1-\xi) \pi .
$$

Lemma 1.2 [15]. If $f(z) \in C$ and $g(z) \in K$, then $f(z)^{*} g(z) \in K$.

\section{Main results}

Theorem 2.1. Let $f_{i}(z) \in S^{*}, g_{i}(z) \in C^{*}$ and $h_{i}(z) \in R_{k}(\xi)$ with $0 \leq \xi<1, k \geq 2$ for all 1 $\leq i \leq n$ If

$$
\sum_{i=1}^{n}\left(\alpha_{i}+\left(\frac{k}{2}-1\right)(1-\rho) \beta_{i}\right) \leq 1,
$$

then integral operator defined by (1.3) belongs to the class of close-to-convex functions.

Proof. Let $f_{i}(z) \in S^{*}$ and $g_{i}(z) \in C^{*}$. Then there exists $\phi_{i}(z) \in C$ such that

$$
f_{i}(z)=z \varphi_{i}^{\prime}(z) .
$$

Now consider

$$
f_{i}(z) * g_{i}(z)=z \varphi_{i}^{\prime}(z) * g_{i}(z)=\varphi_{i}(z) * z g_{i}^{\prime}(z) .
$$

Since $g_{i}(z) \in C^{*}$, then by Alexander-type relation $z g_{i}^{\prime}(z) \in K$. So, by Lemma 1.2, we have 


$$
\varphi_{i}(z) * z g_{i}^{\prime}(z) \in K
$$

which implies that

$$
f_{i}(z) * g_{i}(z) \in K
$$

and hence, by using (1.1),

$$
\int_{\theta_{1}}^{\theta_{2}} \operatorname{Re}\left\{1+\frac{z\left(f_{i}(z) * g_{i}(z)\right)^{\prime \prime}}{\left(f_{i}(z) * g_{i}(z)\right)^{\prime}}\right\} d \theta>-\pi
$$

From (1.3), we obtain

$$
I_{n}\left(f_{i}, g_{i}, h_{i}\right)^{\prime}(z)=\prod_{i=1}^{n}\left(\left(f_{i}(z) * g_{i}(z)\right)^{\prime}\right)^{\alpha_{i}}\left(\frac{h_{i}(z)}{z}\right)^{\beta_{i}}
$$

Differentiating (2.3) logarithmically, we have

$$
\begin{aligned}
1+\frac{I_{n}\left(f_{i}, g_{i}, h_{i}\right)^{\prime \prime}(z)}{I_{n}\left(f_{i}, g_{i}, h_{i}\right)^{\prime}(z)} & =\sum_{i=1}^{n} \alpha_{i} \frac{z\left(f_{i}(z) * g_{i}(z)\right)^{\prime \prime}}{\left(f_{i}(z) * g_{i}(z)\right)^{\prime}}+\sum_{i=1}^{\mathrm{n}} \beta_{i}\left(\frac{z h_{i}^{\prime}(z)}{h_{i}(z)}-1\right)+1 \\
& =\sum_{i=1}^{n} \alpha_{i}\left(1+\frac{z\left(f_{i}(z) * g_{i}(z)\right)^{\prime \prime}}{\left(f_{i}(z) * g_{i}(z)\right)^{\prime}}\right)+\sum_{i=1}^{\mathrm{n}} \beta_{i}\left(\frac{z h_{i}^{\prime}(z)}{h_{i}(z)}\right)+1-\sum_{i=1}^{\mathrm{n}}\left(\alpha_{i}+\beta_{i}\right)
\end{aligned}
$$

Taking real part and then integrating with respect to $\theta$, we get

$$
\begin{aligned}
\int_{\theta_{1}}^{\theta_{2}} \operatorname{Re}\left\{1+\frac{I_{n}\left(f_{i}, g_{i}, h_{i}\right)^{\prime \prime}(z)}{I_{n}\left(f_{i}, g_{i}, h_{i}\right)^{\prime}(z)}\right\} d \theta & =\sum_{i=1}^{n} \alpha_{i} \int_{\theta_{1}}^{\theta_{2}} \operatorname{Re}\left(1+\frac{z\left(f_{i}(z) * g_{i}(z)\right)^{\prime \prime}}{\left(f_{i}(z) * g_{i}(z)\right)^{\prime}}\right) d \theta \\
& +\sum_{i=1}^{\mathrm{n}} \beta_{i} \int_{\theta_{1}}^{\theta_{2}} \operatorname{Re}\left(\frac{z h_{i}^{\prime}(z)}{h_{i}(z)}\right) d \theta+\left(1-\sum_{i=1}^{\mathrm{n}}\left(\alpha_{i}+\beta_{i}\right)\right)\left(\theta_{2}-\theta_{1}\right)
\end{aligned}
$$

Using (2.2) and Lemma 1.1, we have

$$
\begin{aligned}
\int_{\theta_{1}}^{\theta_{2}} \operatorname{Re}\left\{1+\frac{I_{n}\left(f_{i}, g_{i}, h_{i}\right)^{\prime \prime}(z)}{I_{n}\left(f_{i}, g_{i}, h_{i}\right)^{\prime}(z)}\right\} d \theta>-\pi \sum_{i=1}^{n}\left(\alpha_{i}+\left(\frac{k}{2}-1\right)(1-\rho) \beta_{i}\right) \\
+\left(1-\sum_{i=1}^{\mathrm{n}}\left(\alpha_{i}+\beta_{i}\right)\right)\left(\theta_{2}-\theta_{1}\right)
\end{aligned}
$$

From (2.1), we can easily write

$$
\sum_{i=1}^{n}\left(\alpha_{i}+\beta_{i}\right)<\sum_{i=1}^{n}\left(\alpha_{i}+\left(\frac{k}{2}-1\right)(1-\rho) \beta_{i}\right) \leq 1 .
$$

This implies that

$$
\sum_{i=1}^{n}\left(\alpha_{i}+\beta_{i}\right)<1
$$


so, minimum is for $\theta_{1}=\theta_{2}$, we obtain

$$
\int_{\theta_{1}}^{\theta_{2}} \operatorname{Re}\left\{1+\frac{I_{n}\left(f_{i}, g_{i}, h_{i}\right)^{\prime \prime}(z)}{I_{n}\left(f_{i}, g_{i}, h_{i}\right)^{\prime}(z)}\right\} d \theta>-\pi,
$$

and this implies that $I_{n}\left(f_{i} g_{i} h_{i}\right)(z) \in K$.

For $k=2$ in Theorem 2.1, we obtain

Corollary 2.3. Let $f_{i}(z) \in S^{*}, g_{i}(z) \in C^{*}$ and $h_{i}(z) \in S^{*}(\xi)$ with $0 \leq \xi<1$, for all $1 \leq i \leq$ n. If

$$
\sum_{i=1}^{n} \alpha_{i} \leq 1
$$

then $I_{n}\left(f_{\dot{v}} g_{\dot{v}} h_{i}\right)(z) \in K$.

Theorem 2.4. Let $f_{i}(z) \in T_{k}$ and $h_{i}(z) \in R_{k}$ for $1 \leq i \leq n$. If $\alpha_{i}, \beta_{i} \geq 0$ such that $\alpha_{i}+$ $\beta_{i} \neq 0$ and

$$
\sum_{i=1}^{n}\left(\frac{k}{2}\left(\alpha_{i}+\beta_{i}\right)-\beta_{i}\right) \leq 1
$$

then $I_{n}\left(f_{i} h_{i}\right)(z)$ defined by (1.4) belongs to the class of close-to-convex functions.

Proof. From (1.4), we have

$$
I_{n}\left(f_{i}, h_{i}\right)^{\prime}(z)=\prod_{i=1}^{n}\left(f_{i}^{\prime}(z)\right)^{\alpha_{i}}\left(\frac{h_{i}(z)}{z}\right)^{\beta_{i}} .
$$

Differentiating (2.5) logarithmically, we have

$$
1+\frac{I_{n}\left(f_{i}, h_{i}\right)^{\prime \prime}(z)}{I_{n}\left(f_{i}, h_{i}\right)^{\prime}(z)}=\sum_{i=1}^{n} \alpha_{i}\left(1+\frac{z f_{i}^{\prime \prime}(z)}{f_{i}^{\prime}(z)}\right)+\sum_{i=1}^{\mathrm{n}} \beta_{i}\left(\frac{z h_{i}^{\prime}(z)}{h_{i}(z)}\right)+1-\sum_{i=1}^{\mathrm{n}}\left(\alpha_{i}+\beta_{i}\right) .
$$

Taking real part and then integrating with respect to $\theta$, we get

$$
\begin{array}{r}
\int_{\theta_{1}}^{\theta_{2}} \operatorname{Re}\left(1+\frac{I_{n}\left(f_{i}, h_{i}\right)^{\prime \prime}(z)}{I_{n}\left(f_{i}, h_{i}\right)^{\prime}(z)}\right) d \theta=\sum_{i=1}^{n} \alpha_{i} \int_{\theta_{1}}^{\theta_{2}} \operatorname{Re}\left(1+\frac{z f_{i}^{\prime \prime}(z)}{f_{i}^{\prime}(z)}\right) d \theta+\sum_{i=1}^{\mathrm{n}} \beta_{i} \int_{\theta_{1}}^{\theta_{2}} \operatorname{Re}\left(\frac{z h_{i}^{\prime}(z)}{h_{i}(z)}\right) d \theta \\
+\left(1-\sum_{i=1}^{\mathrm{n}}\left(\alpha_{i}+\beta_{i}\right)\right)\left(\theta_{2}-\theta_{1}\right) . \\
>-\frac{k \pi}{2} \sum_{i=1}^{n} \alpha_{i}-\left(\frac{k}{2}-1\right) \pi \sum_{i=1}^{\mathrm{n}} \beta_{i}+\left(1-\sum_{i=1}^{\mathrm{n}}\left(\alpha_{i}+\beta_{i}\right)\right)\left(\theta_{2}-\theta_{1}\right),
\end{array}
$$

where we have used Lemma 1.1 and (1.2)

$$
=-\sum_{i=1}^{n}\left(\left(\frac{k}{2}\right)\left(\alpha_{i}+\beta_{i}\right)-\beta_{i}\right)+\left(1-\sum_{i=1}^{n}\left(\alpha_{i}+\beta_{i}\right)\right)\left(\theta_{2}-\theta_{1}\right) .
$$

From (2.4), we can obtain

$$
\sum_{i=1}^{n}\left(\alpha_{i}+\beta_{i}\right)<1
$$


So minimum is for $\theta_{1}=\theta_{2}$, thus we have

$$
\int_{\theta_{1}}^{\theta_{2}} \operatorname{Re}\left(1+\frac{I_{n}\left(f_{i}, h_{i}\right)^{\prime \prime}(z)}{I_{n}\left(f_{i}, h_{i}\right)^{\prime}(z)}\right) d \theta>-\pi .
$$

This implies that $\mathrm{I}_{\mathrm{n}}\left(f_{i}, h_{i}\right)(z) \in K$.

For $k=2$ in Theorem 2.4, we obtain the following result.

Corollary 2.5. Let $f_{i}(z) \in K, h_{i}(z) \in S^{*}$ for $1 \leq i \leq n$ and

$$
\sum_{i=1}^{n}\left(\frac{k}{2}\left(\alpha_{i}+\beta_{i}\right)-\beta_{i}\right) \leq 1
$$

then $I_{n}\left(f_{i} h_{i}\right)(z)$ defined by (1.4) belongs to the class of close-to-convex functions.

\section{Acknowledgements}

The authors would like to thank the reviewers and editor for improving the presentation of this article, and they also thank Dr. Ihsan Ali, Vice Chancellor AWKUM, for providing excellent research facilities in AWKUM.

\section{Author details}

${ }^{1}$ Department of Mathematics, Abdul Wali Khan University, Mardan, Pakistan ${ }^{2}$ Department of Mathematics, COMSATS

Institute of Information Technology, Islamabad, Pakistan

\section{Authors' contributions}

MA completed the main part of this article, KIN presented the ideas of this article, FG participated in some results of this article. MA made the text file and all the communications regarding the manuscript. All authors read and approved the final manuscript.

\section{Competing interests}

The authors declare that they have no competing interests.

Received: 15 September 2011 Accepted: 19 January 2012 Published: 19 January 2012

\section{References}

1. Kaplan, W: Close-to-convex Schlicht functions. Michigan J Math. 1, 169-185 (1952)

2. Padmanabhan, $K$, Parvatham, R: Properties of a class of functions with bounded boundary rotation. Ann Polon Math. $31,311-323(1975)$

3. Noor, Kl: On radii of convexity and starlikeness of some classes of analytic functions. Int J Math Math Sci. 14(4), 741-746 (1991). doi:10.1155/S016117129100100X

4. Noor, Kl: On some integral operators for certain families of analytic function. Tamkang J Math. 22, 113-117 (1991)

5. Noor, Kl, Haq, W, Arif, M, Mustafa, S: On bounded boundary and bounded radius rotations. J Inequal Appl 2009, 12 (2009). Article ID 813687

6. Noor, Kl: On generalization of close-to-convexity. Int J Math Math Sci. 15(2), 279-290 (1992). doi:10.1155/ S016117129200036X

7. Bulut, S: A new general integral operator defined by Al-Oboudi differential operator. J Inequal Appl 2009, 13 (2009). Article ID 158408

8. Malik, B: On some integral operators for certain classes of P-valent functions. Int J Math Math Sci 2011, 10 (2011). Article ID 783084

9. Frasin, BA: Order of convexity and univalency of general integral operator. J Franklin Inst. 348(6), 1013-1019 (2011). doi:10.1016/j.jfranklin.2011.03.006

10. Breaz, D, Breaz, N: Two integral operator. pp. 13-19. Studia Universitatis Babes-Bolyai, Mathematica, Clunj-Napoca3, (2002)

11. Breaz, D, Owa, S, Breaz, N: A new integral univalent operator. Acta Univ Apulensis Math Inf. 16, 11-16 (2008)

12. Alexander, JW: Functions which map the interior of the unit circle upon simple regions. Ann Math. 17, 12-22 (1916)

13. Miller, SS, Mocanu, PT, Read, MO: Starlike integral operators. Pacific J Math. 79, 157-168 (1978)

14. Noor, Kl: On subclasses of close-to-convex functions of higher order. Int J Math Math Sci. 6(2), 327-334 (1983). doi:10.1155/S0161171283000289

15. Ruscheweyh, S, Sheil-Small, T: Hademard product s of Schlicht functions and the Polya-Schoenberg conjecture Comment Math Helv. 48, 119-135 (1973). doi:10.1007/BF02566116

doi:10.1186/1029-242X-2012-13

Cite this article as: Arif et al: Some properties of an integral operator defined by convolution. Journal of Inequalities and Applications 2012 2012:13. 